\title{
EXPANSION, PERSPECTIVES, AND CHALLENGES
}

\author{
LARS VILHUBER
}

Managing Editor, Journal of Privacy and Confidentiality, and Cornell University e-mail address: managing-editor@journalprivacyconfidentiality.org

\begin{abstract}
The present issue heralds a new phase for the Journal. In our ongoing efforts to provide a forum for a diverse community interested in privacy and confidentiality, we welcome a large expansion of the editorial board. We also introduce not one, but two new sections to the Journal. Perspectives are shorter articles, possibly somewhat opiniated, typically clustered around a same theme. The section on Privacy Challenges encompasses articles that describe practical implementations of privacy-preserving algorithms from a variety of competitions, starting with the NIST PSCR Challenge from 2018-2019. As every year, we also publish select contributions from the Workshop on Theory and Practice of Differential Privacy, which in 2019 was held in London, UK as part of the ACM Conference on Computer Security (CCS).
\end{abstract}

\section{Expansion of the Editorial BoArd}

The Journal has had a relatively small editorial board for a long time. This has proven to be limiting both in our ability to efficiently shepherd articles through the editorial process, as well as in the diversity of specializations present on the board. Over the past six months, we have therefore undertaken an expansion of the editorial board, greatly broadening the expertise that we collectively can marshall, with both greater regional and thematic diversity. We welcome to the board the following new editors (in alphabetical order):

- Stefan Bender, Deutsche Bundesbank, Germany

- Kamalika Chaudhuri, UC San Diego, USA

- Jörg Drechsler, Institut für Arbeitsmarkt- und Berufsforschung, Germany

- Abraham Flaxman, University of Washington, USA

- Daniel L. Goroff, Alfred P. Sloan Foundation, USA

- Daniel Kifer, Penn State University, USA

- Aleksandra Korolova, University of Southern California, USA

- Frauke Kreuter, University of Maryland, USA \& Universität Mannheim, Germany

- Moni Naor, Weizmann Institute of Science, Israel

- Ian Schmutte, University of Georgia, USA

- Aleksandra Slavković, Penn State University, USA

- Adam D. Smith, Boston University, USA

- Kunal Talwar, Apple, USA

Key words and phrases: perspectives, privacy, challenge, synthetic data.

$\int_{\text {Confidentiality }}^{P_{\text {rivacy }}}$

www.journalprivacyconfidentiality.org DOI:10.29012/jpc.749
(C) L. Vilhuber

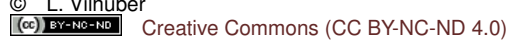


We have also formalized our arrangement with the organizers of the Workshop on Theory and Practice of Differential Privacy, who become ex-officio members of the editorial board in the years surrounding their turn chairing the Workshop. Currently serving are Aleksandar Nikolov, University of Toronto, Michael Hay, Colgate University, and Rachel Cummings, Columbia University.

\section{A New type of ARticle: Perspectives}

With this issue, we are introducing a new type of article: "Perspectives." Articles in this section are meant to provide a view - a perspective - on a particular topic from a more personal point of view, discussing ideas, projects, events, or institutions. The articles do not necessarily reflect the opinions of the editorial board, may be opinionated, but should remain balanced, or counter-balanced within a selection of such articles. They are subject to peer-review, but with a focus on clarity of exposition. Generally, they are meant to be much shorter (5-7 pages), and do not contain much technical notation (though they should contain adequate documentation of their sources). The journal's editors will generally solicit these perspectives, but unsolicited submissions are considered.

We start this section off in this and the next issue with articles emanating from the October 2020 Canadian Research Data Centre Network (CRDCN) conference celebrating the 20th anniversary of the network. ${ }^{1}$ Two sessions focused on retrospective and prospective views on privacy and access. The first session, on "Microdata Access: Perspectives From Canada, France, the UK and the US," moderated by the author of this editorial, had participants reflect on the history of international data access mechanisms in connection with the history of the Canadian network. ${ }^{2}$ Ron Jarmin's reflections on the similarities and differences between the US and Canadian network are included in this issue, and Roxane Silberman's reflections on the French network will be included in the next issue. Felix Ritchie reflects on the topic of the second session on "Privacy and Microdata Access: Two Worlds Colliding?" Michael Harvey's perspective on data access from a privacy regulator's point of view, from the same session, will appear in the next issue.

The author of these lines edited the present selection of perspectives.

\section{A new ReCurring topic: Privacy Challenges}

Another new section is dedicated to "privacy challenges." Due to the differences between algorithms on paper and bits in motion, it is not always easy to tell what is the state-of-the-art from a practitioner's perspective. In this section, we will be featuring articles that describe and analyze successful techniques for protecting privacy in challenge datasets. We start with an article by Claire McKay Bowen and Joshua Snoke, reporting on various algorithms from the 2018-2019 National Institute of Standards and Technology Public Safety Communications Research (NIST PSCR) Division's "Differential Privacy Synthetic Data Challenge." Future issues will have individual participant teams from that and other competitions going into much detail about their entries to the challenges. Daniel Kifer will edit this section.

\footnotetext{
${ }^{1}$ See https://www.crdcn20.ca/ for more information.

${ }^{2}$ The history of the Canadian network was described extensively in Currie and Fortin (2015).
} 
4. TPDP 2019

The 5th Workshop on Theory and Practice of Differential Privacy was held in London, UK on 11 November 2019 as part of the ACM Conference on Computer Security (CCS). The workshop brought together researchers from computer science and statistics to discuss recent developments in both the theory and practice of differential privacy. This issue presents the following two contributions from that workshop:

- Albert Cheu, Adam Smith, and Jonathan Ullman discuss "Manipulation Attacks in Local Differential Privacy," and conclude with a caution when deploying local differential privacy.

- Adam Sealfon and Jonathan Ullman present an "optimal, computationally efficient, private algorithm for estimating the edge-density" under certain conditions in "Efficiently Estimating Erdős-Rényi Graphs with Node Differential Privacy."

Michael Hay edited the TPDP 2019 papers.

\section{REFERENCES}

R.F. Currie and S. Fortin. Social Statistics Matter: A History of the Canadian RDC Network. Canadian Research Data Centre Network = Réseau canadien des Centres de données de recherche, 2015. ISBN 978-0-9947581-1-8. http://rdc-cdr . $\mathrm{ca} / \mathrm{sites} / \mathrm{default/files/social-statistics-matter-crdcn-history.pdf.}$ 